\title{
Molecular Targets and Associated Signaling Pathways of Jingshu Granules in Ovarian Cysts Based on Systemic Pharmacological Analysis
}

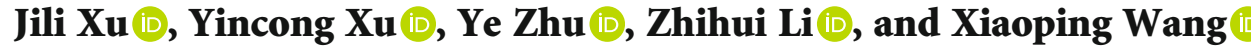 \\ College of Traditional Chinese Medicine, Shandong University of Traditional Chinese Medicine, Jinan City, Shandong Province, \\ 250355, China
}

Correspondence should be addressed to Xiaoping Wang; jnwxp1962@163.com

Received 16 November 2020; Accepted 9 January 2021; Published 21 January 2021

Academic Editor: Ahmet Özer Sehirli

Copyright (C) 2021 Jili Xu et al. This is an open access article distributed under the Creative Commons Attribution License, which permits unrestricted use, distribution, and reproduction in any medium, provided the original work is properly cited.

\begin{abstract}
Background. More than a third of women could develop ovarian cysts during their lifetime. Jingshu granules are used for the treatment of gynecological disease of primary dysmenorrhea. However, the molecular mechanisms of Jingshu granules in ovarian cysts are still unreported. We aimed to find the active ingredients, molecular targets, and potential signaling pathways of Jingshu granules in ovarian cysts by using the systemic pharmacological analysis. Methods. Firstly, the effect of Jingshu granules on female hormones and reproductive organs of young female rats was evaluated. Secondly, candidate pharmaceutical ingredients of Jingshu granules were retrieved from the traditional Chinese medicine systems pharmacology (TCMSP) database and analysis platform. Potential protein targets for the active ingredients in Jingshu granules were then identified according to the oral bioavailability and drug-likeness indices. Thirdly, ovarian cyst-related gene targets were screened based on different databases. Finally, enrichment analysis was used to analyze the potential biological function of intersection targets between Jingshu granules and ovarian cysts. Results. In young female rats, Jingshu granules reduced the secretion of estradiol, progesterone, and prolactin and could affect the development of the uterus. This suggested that Jingshu granules played roles in hormone secretion and reproduction. From the TCMSP, a total of 1021 pharmaceutical ingredients of Jingshu granules were retrieved. After further screening, a total of 166 active ingredients and 159 protein targets of Jingshu granules were identified. In addition, 4488 gene targets of ovarian cysts were screened out. After taking the intersection, a total of 110 intersection targets were identified between potential protein targets of Jingshu granules and gene targets of ovarian cysts. In the functional analysis of 110 intersection targets, 8 signaling pathways including progesterone-mediated oocyte maturation (MAPK8 and CDK1 involved), GnRH signaling pathway (JUN involved), T cell receptor signaling pathway and Toll-like receptor signaling pathway (MAPK1 involved), NOD-like receptor signaling pathway (TNF, IL6, and IL1B involved), p53 signaling pathway (CDK2 and CDK4 involved), VEGF signaling pathway (MAPK14 involved), and PPAR signaling pathway (PPARG involved) were obtained. Conclusion. Our study revealed that Jingshu granules could function in patients with ovarian cysts through a number of molecular targets and signaling pathways. Our study may provide a new field into the mechanisms of Jingshu granules in ovarian cysts, from the molecular to the signaling pathway level.
\end{abstract}

\section{Introduction}

An ovarian cyst is a fluid-containing sac ( $>20 \mathrm{~mm}$ in diameter) that is formed in the ovaries. It is common in women of all ages, and its prevalence in pre- and postmenopausal women is $35 \%$ and $17 \%$, respectively [1]. An ovarian cyst consists of five types, including follicular cysts, lutein cysts, cystic corpora lutea, cystic rete ovarii, and cysts of subsurface epithelial structures [2-4]. The most common symptoms of these cysts are dyspareunia, chronic pelvic pain, and gastrointestinal and urinary symptoms [5, 6]. Endometriosis, serous, mucinous, teratomas, inflammation, Brenner, and fibroma-thecoma are benign ovarian cysts, which result from the unruptured follicle. However, ovarian cysts (cysts larger than $40 \mathrm{~mm}$ and abnormal serum CA-125 levels) with intolerable symptoms may form the malignant lesion, such as the 
cystadenocarcinoma or cystadenoma $[6,7]$. At this time, surgical removal of the cysts is required. However, the removal of ovarian cysts is also related to injury to the ovarian reserve $[8,9]$. The pathology of ovarian cysts is complex. It is found that age, diet, the last menstrual period, chemotherapy, duration of tamoxifen, and serum levels of estrogen and folliclestimulating hormone are associated with the occurrence of ovarian cysts $[10,11]$.

The traditional Chinese medicine (TCM) formula, composed of various active ingredients, has a complex action mechanism, which could be related to multiple targets and pathways. Recently, TCM has attracted remarkable attention in female reproductive dysfunctions in view of its safety and efficacy [12-16]. For example, Bushen Tianjing Recipe has been used for premature ovarian failure [17]. Jingshu granules consist of 11 herbs, including Angelica, radix Paeoniae alba, radix Bupleuri, nutgrass Galingale rhizome, radix Curcumae, rhizoma Ligustici wallichii, Corydalis tuber, Ginseng, rhizoma Atractylodis macrocephalae, dodder weed, and licorice. The water decoction of Jingshu granules has been in clinical application for more than 50 years and has a significant effect on the treatment of primary dysmenorrhea. However, few studies reported the action mechanism of Jingshu granules in other gynecological diseases, such as ovarian cysts. In this study, we tried to explore the action mechanisms of Jingshu granules, which could provide a basis for the understanding of Jingshu granules in the treatment of ovarian cysts.

\section{Methods}

2.1. The Effect of Jingshu Granules on Female Hormones and Reproductive Organs of Young Female Rats. In order to study the preliminary effect of Jingshu granules on female hormones and reproduction organs, 40 young SPF SpragueDawley (SD) female rats (sexually mature and unmated) aged 12 weeks old $(240 \pm 20 \mathrm{~g})$ were selected in this study. These rats were purchased from Jinan Pengyue Experimental Animal Breeding Co., Ltd. (License No.: SCXK (Lu) 20180003). Animal experiments were conducted in the barrier environment facility of the Experimental Animal Center of Shandong University of Traditional Chinese Medicine (License Number: SYXK (Lu) 20170022) at room temperature of 23 $\pm 1^{\circ} \mathrm{C}$ and humidity at $45 \pm 5 \%$ with the fluorescent light irradiation. These rats were fed with Co60 maintenance feed (feed production license no.: SCXK (Beijing) 20190003) and sterile water. They were randomly divided into four groups. In this study, the Jingshu granules are purchased from Shandong Hospital of Traditional Chinese Medicine. In the first group, the rats were orally given a high dose of Jingshu granules $(15.2 \mathrm{~g} / \mathrm{kg})$, which was equivalent to 10 times the clinical dosage of the human clinical dose. In the second group, the rats were orally given a medium dose of Jingshu granules $(7.6 \mathrm{~g} / \mathrm{kg})$, which was equivalent to 5 times the clinical dosage of the human clinical dose. The rats in the third group received a low dose of Jingshu granules $(3.8 \mathrm{~g} / \mathrm{kg})$, which was equivalent to 2.5 times the human clinical dose. The rats in the fourth group were given an equal volume of water as the normal control. After continuous gavage for 30 days,
$45 \mathrm{mg} / \mathrm{kg}$ sodium pentobarbital was injected intraperitoneally for anesthesia. Blood samples were collected from the inferior vena cava to measure progesterone, estradiol, and prolactin. The uterus and ovaries were dissected and weighed. All animal studies were approved by the Shandong University of Traditional Chinese Medicine (SDUTCM20181102001) and were in accordance with the Guidelines for Care and Use of Experimental Animals.

2.2. Identification of Active Ingredients and Protein Targets of Jingshu Granules. In order to explore the drug target of Jingshu granules, systemic pharmacological analysis was performed. All compounds of eleven herbs in Jingshu granules were retrieved from the TCM systems pharmacology (TCMSP) (http://lsp.nwu.edu.cn/tcmsp.php) database. The TCMSP includes information on all 500 TCM formulae [18]. Molecules with oral bioavailability $(\mathrm{OB}) \geq 30 \%$ and drug-likeness $(\mathrm{DL}) \geq 0.18$ were considered active ingredients of Jingshu granules. The potential protein targets for the active ingredients in Jingshu granules were further identified by using the systematic drug targeting approach [19].

2.3. Identification of Ovarian Cyst-Related Gene Targets. In order to investigate the molecular mechanism of Jingshu granules in the hormones and reproduction-related diseases of ovarian cysts, the gene targets of ovarian cysts were retrieved from the therapeutic target database (TTD, http:// db.idrblab.net/ttd/), DrugBank (https://www.drugbank.ca/), DisGeNET (https://www.disgenet.org/home/), Comparative Toxicogenomics Database (CTD, https:/ctdbase.org/ ;jessionid=0703B75089B9C2C2727D48FD74B43FA9), and MalaCards (https://www.malacards.org/). Only those intersection targets between potential protein targets for the active ingredients in Jingshu granules and gene targets of ovarian cysts were used for subsequent analysis.

2.4. Functional Analysis of Intersection Targets between Jingshu Granules and Ovarian Cysts. To further understand the potential biological function of intersection targets between Jingshu granules and ovarian cysts, Gene Ontology (GO) and Kyoto Encyclopedia of Genes and Genomes (KEGG) signaling pathway enrichment were performed via the DAVID database. The criterion for selecting significantly enriched functional terms of intersection targets was $p<0.05$

2.5. Statistical Analysis. Statistical calculation was performed using SPSS software (SPSS Inc., Chicago, IL, USA). Comparisons between two groups were assessed using Student's unpaired $t$-tests. A level of $p<0.05$ was selected as the statistical significance in every comparison.

\section{Results}

3.1. The Effect of Jingshu Granules on Female Hormones and Reproductive Organs of Young Female Rats. From the result of Jingshu granules on female hormones of young female rats (Table 1), we found that the high, medium, and low doses of Jingshu granules significantly reduced the expression of prolactin. The high and medium doses of Jingshu granules 
TABLE 1: The effect of Jingshu granules on female hormones of young female rats.

\begin{tabular}{lcccc}
\hline Group & Dose $(\mathrm{g} / \mathrm{kg})$ & Estradiol $(\mathrm{pg} / \mathrm{ml})$ & Progesterone $(\mathrm{ng} / \mathrm{ml})$ & Prolactin $(\mathrm{ng} / \mathrm{ml})$ \\
\hline High & 15.2 & $27.8 \pm 15.2^{* *}$ & $0.58 \pm 0.16^{* *}$ & $2.45 \pm 1.28^{* *}$ \\
Medium & 7.6 & $30.3 \pm 8.9^{*}$ & $0.74 \pm 0.20$ & $5.27 \pm 1.62^{* *}$ \\
Low & 3.8 & $37.5 \pm 8.2$ & $0.94 \pm 0.85$ & $6.63 \pm 2.84^{* *}$ \\
Normal control & Equal volume of water & $42.9 \pm 11.7$ & $0.92 \pm 0.24$ & $9.62 \pm 1.29$ \\
\hline
\end{tabular}

The result was represented as mean $\pm \mathrm{SD} .{ }^{*} p<0.05,{ }^{* *} p<0.01$ compared with the normal control.

TABLE 2: The effect of Jingshu granules on the reproductive organs of young female rats.

\begin{tabular}{lcccc}
\hline Group & Dose $(\mathrm{g} / \mathrm{kg})$ & Body weight $(\mathrm{g})$ & Uterus coefficient $(\mathrm{mg} / 100 \mathrm{~g})$ & Ovary coefficient $(\mathrm{mg} / 100 \mathrm{~g})$ \\
\hline High & 15.2 & $180.0 \pm 15.7$ & $28.6 \pm 6.4^{*}$ & $35.7 \pm 7.9$ \\
Medium & 7.6 & $186.6 \pm 10.5$ & $32.2 \pm 8.0$ & $36.8 \pm 5.0$ \\
Low & 3.8 & $185.2 \pm 15.9$ & $39.9 \pm 9.2$ & $45.0 \pm 7.9$ \\
Normal control & Equal volume of water & $193.3 \pm 21.6$ & $39.3 \pm 10.4$ & $39.8 \pm 5.6$ \\
\hline
\end{tabular}

The result was represented as mean \pm SD. ${ }^{*} p<0.05$ compared with the normal control.

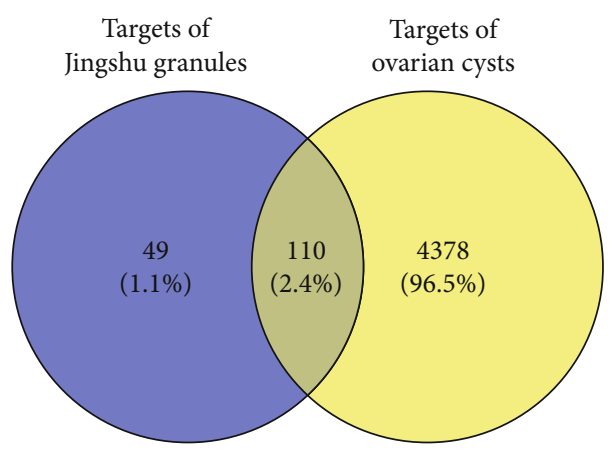

FIGURE 1: Venn diagram of targets between potential protein targets of Jingshu granules and gene targets of ovarian cysts.

remarkably reduced the expression of estradiol. In addition, the large dose group of Jingshu granules had a significant weakening effect on progesterone. This suggested that Jingshu granules could affect the secretion of female hormones. From the result of Jingshu granules on the reproductive organs of young female rats (Table 2), we found that the medium and low doses of Jingshu granules had no significant effect on the body weight and sexual organ weight of the young female rats. The high dose of Jingshu granules had no significant effect on the body weight and ovarian weight of the young female rats. However, a high dose of Jingshu granules could reduce the uterine weight of young female rats. This indicated that Jingshu granules could affect the development of the uterus.

3.2. Intersection Targets between Potential Protein Targets of Jingshu Granules and Gene Targets of Ovarian Cysts. Clinically, an ovarian cyst is a hormone- and reproductionrelated disease. In view of the roles of Jingshu granules in hormone secretion and reproduction, we tried to investigate the molecular mechanism of Jingshu granules in ovarian cysts based on systemic pharmacological analysis. From the
TCMSP, a total of 1021 pharmaceutical ingredients of Jingshu granules were retrieved. After further screening under the threshold of oral bioavailability $(\mathrm{OB}) \geq 30 \%$ and druglikeness $(\mathrm{DL}) \geq 0.18$, a total of 166 active ingredients and 159 protein targets of Jingshu granules were identified. In the TTD, DrugBank, DisGeNET, Comparative Toxicogenomics Database, and MalaCards, we found 4488 gene targets of ovarian cysts. After taking the intersection, a total of 110 intersection targets between potential protein targets of Jingshu granules and gene targets of ovarian cysts were identified (Figure 1). These 110 intersection targets were targeted by 165 active ingredients in 11 herbs of Jingshu granules. 165 active ingredients were from 9 in radix Paeoniae alba, 4 in rhizoma Atractylodis macrocephalae, 14 in radix Bupleuri, 7 in rhizoma Ligustici wallichii, 2 in Angelica, 58 in licorice, 19 in Ginseng, 9 in dodder weed, 17 in nutgrass Galingale rhizome, 49 in Corydalis tuber, and 4 in radix Curcumae. Among which, 10 active ingredients were found in two or more herbs.

3.3. Functional Analysis of Intersection Targets between Jingshu Granules and Ovarian Cysts. In order to understand the potential biological function of intersection targets between Jingshu granules and ovarian cysts, GO and KEGG signaling pathway enrichment analysis was performed. The top 15 enriched GO terms and KEGG pathways are shown in Figures 2 and 3, respectively. In addition, all KEGG signaling pathways and involved genes are listed in Table 3 in detail. Among which, 8 signaling pathways were identified, such as progesterone-mediated oocyte maturation (MAPK8 and CDK1 involved), GnRH signaling pathway (JUN involved), $\mathrm{T}$ cell receptor signaling pathway and Toll-like receptor signaling pathway (MAPK1 involved), NOD-like receptor signaling pathway (TNF, IL6, and IL1B involved), p53 signaling pathway (CDK2 and CDK4 involved), VEGF signaling pathway (MAPK14 involved), and PPAR signaling pathway (PPARG involved). Information of the above 11 


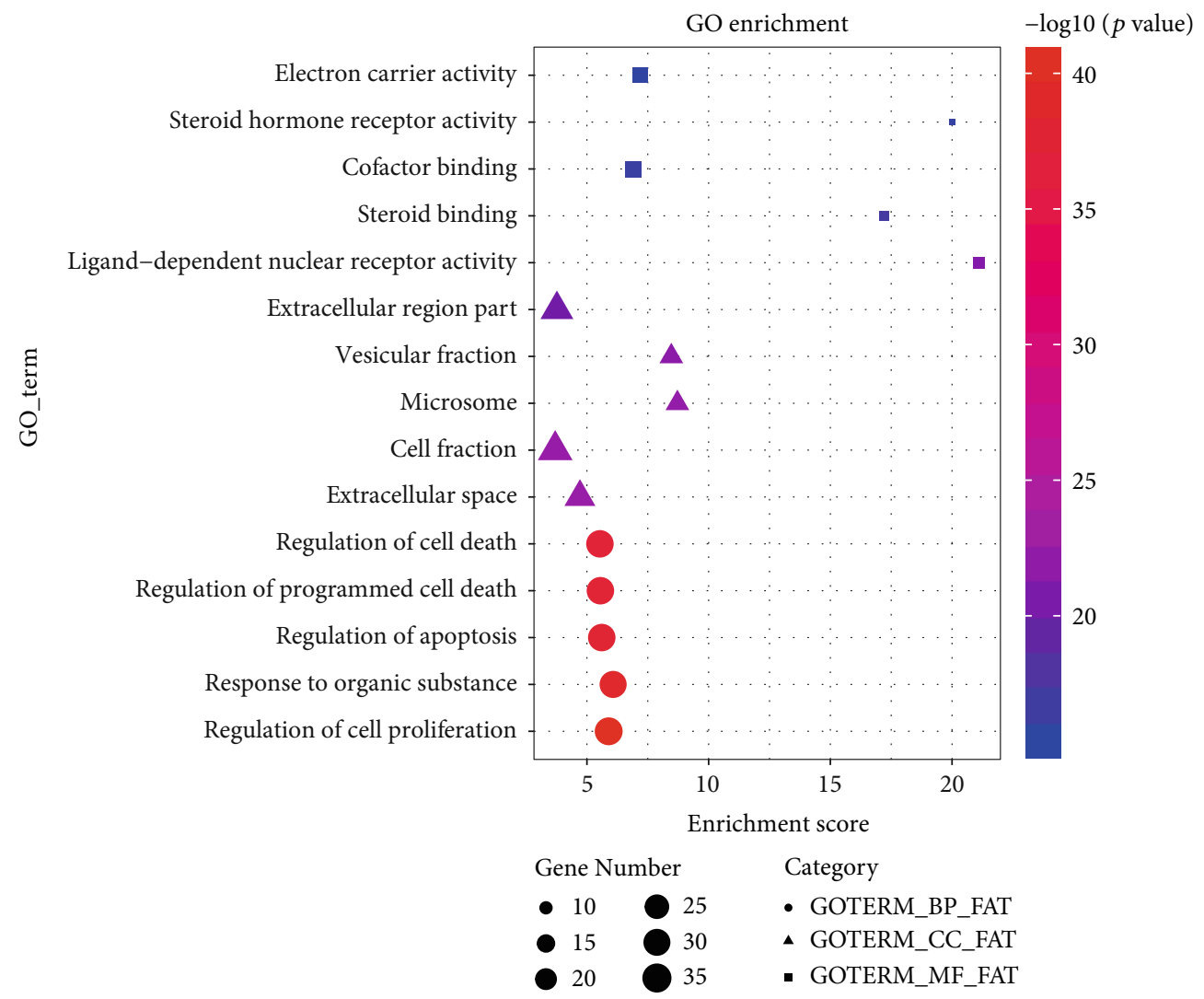

FIGURE 2: Top 15 enriched GO terms of intersection targets between Jingshu granules and ovarian cysts.

involved ovarian cyst-related targets of Jingshu granules is shown in Table 4. Additionally, the network with 11 herbs, 165 active ingredients, 110 intersection targets, and 8 signaling pathways is shown in Figure 4.

\section{Discussion}

Some functional cysts, including cystic follicles and follicular cysts, contain estrogen in the luminal fluid [20]. The detailed mechanism underlying the cystic changes in the ovary remains unknown, although the relationship between ovarian cysts and increased estrogen levels has been found [5]. Knauf et al. found the increased progesterone concentrations in cyst fluid [6]. Prolactin metabolism disorder has been considered one of the pathological mechanisms of multicystic ovarian disease [21]. Perreault et al. proposed that suppression of prolactin secretion could solve some gynecologic problems [22]. Thus, it can be seen that estrogen, increased levels of progesterone, and prolactin are associated with the ovarian cyst. In this study, we found that Jingshu granules could affect the secretion of estradiol, progesterone, and prolactin and affect the development of the uterus of young female rats. This suggested that Jingshu granules played roles in hormone secretion and reproduction, which may be a potential drug for ovarian cyst treatment.

In order to investigate the molecular mechanism of Jingshu granules in the hormones and reproduction-related diseases of ovarian cysts, systemic pharmacological analysis was performed to find active ingredients and protein targets of Jingshu granules and ovarian cyst-related gene targets. Significantly, 110 intersection targets were identified between potential protein targets of Jingshu granules and gene targets of ovarian cysts. According to the KEGG enrichment analysis of 110 intersection targets, 8 signaling pathways including progesterone-mediated oocyte maturation (MAPK8 and CDK1 involved), GnRH signaling pathway (JUN involved), $\mathrm{T}$ cell receptor signaling pathway and Toll-like receptor signaling pathway (MAPK1 involved), NOD-like receptor signaling pathway (TNF, IL6, and IL1B involved), p53 signaling pathway (CDK2 and CDK4 involved), VEGF signaling pathway (MAPK14 involved), and PPAR signaling pathway (PPARG involved) were obtained.

Progesterone could enhance the production of proteolytic enzymes that are important for the follicle rupture at ovulation [23]. In polycystic ovary syndrome, some miRNAs target a number of genes to play different functions, such as progesterone-mediated oocyte maturation [24]. Upregulation of MAPK8 (an apoptosis-related gene) is related to oocyte dysfunction and is involved in various signaling pathways in granulosa cells [25-34]. It is speculated that MAPK8 is one of the therapeutic targets for polycystic ovary syndrome [35]. The CDK1 is an important signaling pathway that is evolutionarily conserved in controlling oocyte growth and meiotic maturation. Kanatsu-Shinohara et al. have demonstrated that CDK1 is an important limiting factor in female gametes [36]. The expression of CDK1 is increased in mature oocytes [37]. In rat oocytes, sustained reduced levels of Thr-161-phosphorylated CDK1 destabilize the 


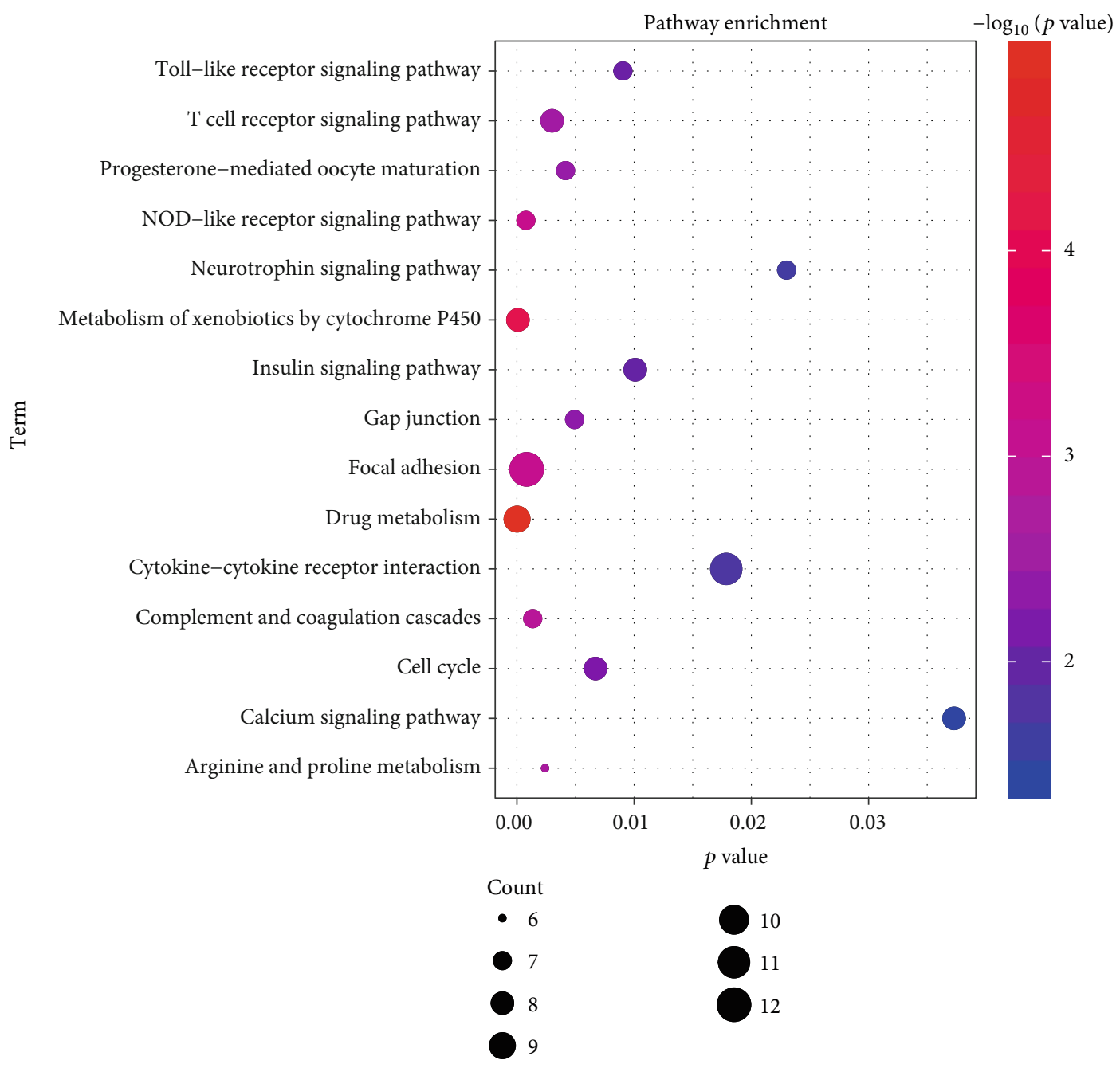

FIGURE 3: Top 15 enriched KEGG signaling pathways of intersection targets between Jingshu granules and ovarian cysts.

maturation-promoting factor [38, 39]. In addition, CDK1null oocytes are permanently arrested at the GV stage, suggesting that CDK1 activity is crucial to the resumption of the first meiosis [40]. Our result indicated that Jingshu granules may exert a medicinal effect by targeting the MAPK8 and CDK1 of the progesterone-mediated oocyte maturation signaling pathway in ovarian cysts.

It is reported that slow GnRH pulse frequencies favor follicle-stimulating hormone production and secretion, whereas rapid frequencies favor luteinizing hormone production and secretion [41-43]. It is worth mentioning that $\mathrm{GnRH}-\mathrm{a}$ is related to the formation of functional ovarian cysts [44]. In the mature gonadotrope cells, GnRH also induces c-JUN transcription, which leads to upregulation of cell cycle proteins [45]. In granulose cells, the conjunction of $c$-JUN and TAF $4 b$ regulates the expression of ovarian genes [46]. In the follicle cells, knockdown of JUN results in engulfment defects in the ovary [47]. It is noted that JUN has been identified as a potential key regulator in the development of polycystic ovary syndrome. Furthermore, decreased levels of p-c-JUN have been found in the ovary tissues of polycystic ovary syndrome rats [48]. Thus, it can be seen that JUN in the GnRH signaling pathway may play an important regulatory role in the ovary. Jingshu granules could be used for ovarian cyst treatment by targeting JUN in the GnRH signaling pathway.

Immune system-related cells ( $\mathrm{T}$ cells) influence the differentiation of primordial follicles and the formation of granulosa cells [49-51]. It has been demonstrated that the absence of regulatory $\mathrm{T}$ cells is a prerequisite for cyst formation in the polycystic ovary syndrome [52]. The expression of innate immune system-related Toll-like receptors 2 and 4 was found in the ovarian cumulus cells and granulosa cells during ovulation [53]. It is found that other Toll-like receptor types are found in regulatory $\mathrm{T}$ cells from patients with endometriosis [54]. MAPK1 is involved in regulating cell proliferation and apoptosis $[55,56]$. The signaling pathway of MEK/MAPK1/3 is crucial to oocyte complex expansion and follicle rupture [57-59]. It is found that MAPK1 is differentially expressed in the endometrium in ovarian endometriosis [60]. In the ovarian tissue of polycystic ovary syndrome patients, MAPK1 could activate autophagy [61]. It is noted that increased expression of MAPK1 had been found in the ovarian cystic epithelium [62]. Herein, we found that MAPK1 was involved in both the $\mathrm{T}$ cell receptor signaling pathway and the Toll-like receptor signaling pathway. This suggested 
TABLE 3: All enriched KEGG signaling pathways of intersection targets between Jingshu granules and ovarian cysts.

\begin{tabular}{|c|c|c|}
\hline Term & $p$ value & Genes \\
\hline $\begin{array}{l}\text { Neuroactive ligand-receptor } \\
\text { interaction }\end{array}$ & $1.75 E-07$ & $\begin{array}{c}\text { OPRM1, DRD1, DRD2, GABRA5, NR3C1, CHRM5, HTR1B, ADRB2, ADRB1, CHRM4, } \\
\text { CHRM3, GRIA2, CHRM2, CHRM1, F2, ADRA1B, ADRA1A, ADRA2C, ADRA2B, } \\
\text { ADRA1D, HTR2A }\end{array}$ \\
\hline Calcium signaling pathway & $2.47 E-06$ & $\begin{array}{c}\text { EGFR, DRD1, CHRM5, ADRB2, ADRB1, CHRM3, CHRM2, CHRM1, ADRA1B, ADRA1A, } \\
\text { NOS3, PRKACA, NOS2, PPP3CA, ADRA1D, HTR2A }\end{array}$ \\
\hline Focal adhesion & 0.002679 & EGFR, MAPK1, JUN, GSK3B, BCL2, MET, VEGFA, COL3A1, MAPK8, COL1A1, EGF, KDR \\
\hline $\begin{array}{l}\text { Cytokine-cytokine receptor } \\
\text { interaction }\end{array}$ & 0.04291 & EGFR, IL6, TNF, CCL2, MET, VEGFA, IFNG, IL1B, EGF, KDR, IL2 \\
\hline MAPK signaling pathway & 0.047776 & EGFR, MAPK1, TNF, MAPK14, JUN, TP53, IL1B, MAPK8, PRKACA, PPP3CA, EGF \\
\hline Gap junction & $7.78 E-05$ & EGFR, CDK1, MAPK1, DRD1, ADRB1, DRD2, GJA1, PRKACA, EGF, HTR2A \\
\hline $\begin{array}{l}\text { T cell receptor signaling } \\
\text { pathway }\end{array}$ & 0.001614 & MAPK1, TNF, MAPK14, JUN, GSK3B, IFNG, PPP3CA, CDK4, IL2 \\
\hline $\begin{array}{l}\text { NOD-like receptor signaling } \\
\text { pathway }\end{array}$ & $2.53 E-04$ & MAPK1, IL6, TNF, CCL2, MAPK14, IL1B, MAPK8, CASP1 \\
\hline $\begin{array}{l}\text { Progesterone-mediated oocyte } \\
\text { maturation }\end{array}$ & 0.001846 & PGR, CDK1, MAPK1, MAPK14, MAPK8, PRKACA, CCNA2, CDK2 \\
\hline Cell cycle & 0.014242 & CDK1, GSK3B, TP53, CHEK1, RB1, CDK4, CCNA2, CDK2 \\
\hline Drug metabolism & 0.001617 & GSTM1, CYP3A4, CYP2B6, MAOA, MAOB, CYP1A2, GSTP1 \\
\hline $\begin{array}{l}\text { Complement and coagulation } \\
\text { cascades }\end{array}$ & 0.002808 & PLAT, F10, THBD, F3, F2, F7, PLAU \\
\hline VEGF signaling pathway & 0.004269 & MAPK1, PTGS2, MAPK14, VEGFA, NOS3, PPP3CA, KDR \\
\hline Apoptosis & 0.008778 & TNF, BCL2, CASP7, TP53, IL1B, PRKACA, PPP3CA \\
\hline GnRH signaling pathway & 0.015262 & EGFR, MAPK1, MAPK14, JUN, MAPK8, PRKACA, MMP2 \\
\hline $\begin{array}{l}\text { Toll-like receptor signaling } \\
\text { pathway }\end{array}$ & 0.017491 & MAPK1, IL6, TNF, MAPK14, JUN, IL1B, MAPK8 \\
\hline Oocyte meiosis & 0.025501 & PGR, CDK1, MAPK1, AR, PRKACA, PPP3CA, CDK2 \\
\hline $\begin{array}{l}\text { Neurotrophin signaling } \\
\text { pathway }\end{array}$ & 0.042293 & MAPK1, MAPK14, JUN, GSK3B, BCL2, TP53, MAPK8 \\
\hline $\begin{array}{l}\text { Arginine and proline } \\
\text { metabolism }\end{array}$ & 0.004458 & ODC1, GOT1, MAOA, MAOB, NOS3, NOS2 \\
\hline Arachidonic acid metabolism & 0.005652 & AKR1C3, PTGS2, CYP2B6, PTGS1, LTA4H, ALOX5 \\
\hline $\begin{array}{l}\text { Metabolism of xenobiotics by } \\
\text { cytochrome P } 450\end{array}$ & 0.007574 & GSTM1, AKR1C3, CYP3A4, CYP2B6, CYP1A2, GSTP1 \\
\hline Glioma & 0.009285 & EGFR, MAPK1, TP53, RB1, EGF, CDK4 \\
\hline ErbB signaling pathway & 0.033168 & EGFR, MAPK1, JUN, GSK3B, MAPK8, EGF \\
\hline p53 signaling pathway & 0.049093 & CDK1, TP53, CHEK1, CDK4, CDK2 \\
\hline Tryptophan metabolism & 0.048697 & MAOA, MAOB, CYP1A2, CAT \\
\hline
\end{tabular}

the role of these signaling pathways in the therapy of ovarian cysts by Jingshu granules.

It is reported that the NOD-like receptor signaling pathway is highly involved in the development of polycystic ovary syndrome [63]. In polycystic ovary syndrome, hypermethylation in TNF contributes to androgen excess [64]. Moreover, TNF suppresses follicle-stimulating hormone-induced activation of the chorionic gonadotropin receptor promoter, making it a key factor contributing to hyperandrogenemia [65]. Interestingly, the expression of TNF- $\alpha$ has been found in serum and peritoneal fluid of women with benign serous ovarian cysts [66]. Brewer and Balen found that IL6 could stimulate the expansion of the cumulus-oocyte complexes and affect oocyte maturation through alterations in steroidogenesis and interaction with metabolic hormones $[67,68]$. It is inferred that the single nucleotide polymorphism in IL6 is a genetic cause of polycystic ovary syndrome [69-71]. IL1B is involved in the regulation of ovarian primordial follicle assembly [72]. It is hypothesized that high expression of IL1B in granulosa cells induces a premature influx of leukocytes and impairs maturation and subsequent ovulation [73]. In the present study, we found that TNF, IL6, and IL1B were all involved in the NOD-like receptor signaling pathway, which may provide a new field for Jingshu granules in the treatment of ovarian cysts.

The presence of a mutation in the gene p53 is common in type II ovarian cancer [66]. CDK2, highly expressed in the ovary libraries, plays a crucial role in controlling follicle cell cycles $[74,75]$. Satyanarayana and Kaldis found that the knockout of CDK2 destroyed the fertility of female mice 
TABLE 4: Information regarding ovarian cyst-related targets of Jingshu granules.

\begin{tabular}{lcc}
\hline Target_ID & Target_name & Gene \\
\hline TAR00431 & Cell division control protein 2 homolog & CDK1 \\
TAR00482 & Cell division protein kinase 2 & CDK2 \\
TAR00573 & Cell division protein kinase 4 & CDK4 \\
TAR00418 & Interleukin-1 beta & IL1B \\
TAR00351 & Interleukin-6 & IL6 \\
TAR00414 & Transcription factor AP-1 & JUN \\
TAR00354 & Mitogen-activated protein kinase 1 \\
TAR00402 & Mitogen-activated protein kinase 14 & MAPK1 \\
TAR00704 & Mitogen-activated protein kinase 8 & MAPK14 \\
TAR00078 & Peroxisome proliferator-activated receptor gamma & MAPK8 \\
TAR00265 & Tumor necrosis factor & PPARG \\
\hline
\end{tabular}

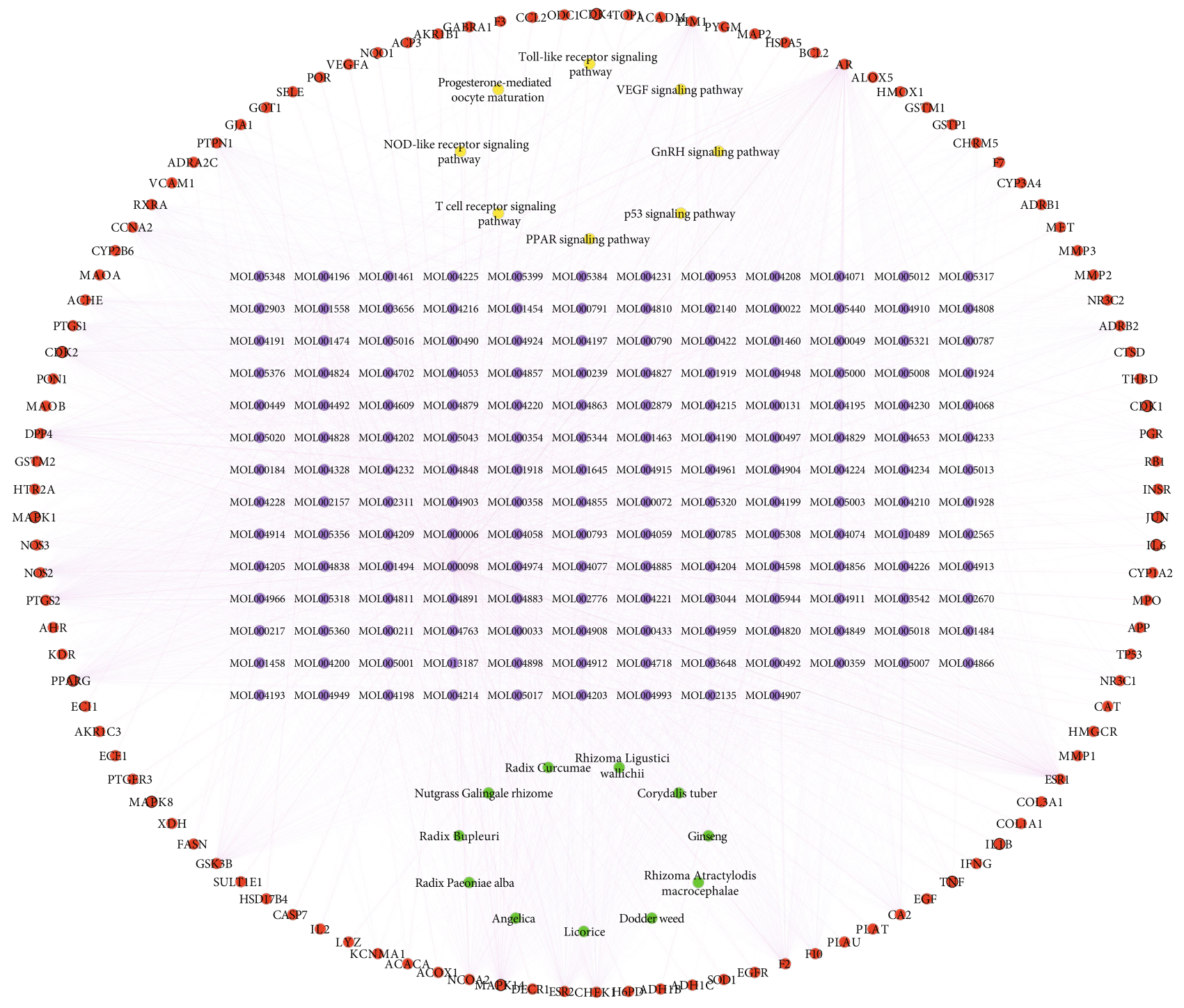

FIGURE 4: The network with 11 herbs, 165 active ingredients, 110 intersection targets, and 8 signaling pathways. Green circle, purple circle, red circle, and yellow circle represented the herb, active ingredient, intersection target, and signaling pathway, respectively. Black circle line represented 11 intersection targets in 8 signaling pathways. 
[76]. CDK4 is associated with follicular growth [77]. In addition, CDK4 is critical for luteal function and granulosa cell proliferation [78]. In this study, we found that both CDK2 and CDK4 were involved in the p53 signaling pathway. This indicated that Jingshu granules may affect the follicle cell cycle in the development of ovarian cysts.

The increased expression of VEGF in the theca layer of the ovary leads to a decrease in ovulation [79-81]. In polycystic ovary syndrome, elevated VEGF prevents granulosa cell apoptosis and follicle atresia, which contribute to the growth and persistence of follicles [82]. However, overexpression of VEGF receptors could be related to the accumulation of follicular fluid and abnormal follicular growth. In addition, VEGF is involved in other reproduction disorders, such as endometriosis [83]. It is found that the luteinizing hormone surge could trigger follicular maturation and ovulation by activating the MAPK14 signaling pathway in granulosa cells $[84,85]$. In addition, MAPK14 plays a role in androgen biosynthesis [86]. The expression of MAPK14 has been found in patients with endometriosis and polycystic ovary syndrome $[86,87]$. Our result suggested that Jingshu granules may promote ovulation by targeting MAPK14 in the VEGF signaling pathway in the therapy of ovarian cysts.

PPAR $\gamma$, a subtype of PPAR, is an important transcription factor associated with follicular differentiation [88]. Downregulation of PPAR $\gamma$ in response to the luteinizing hormone is crucial to ovulation and luteinization [89]. PPARG, regulated by the progesterone receptor, is crucial to follicular maturation and remodeling [90]. In granulosa cells, PPARG is involved in nuclear signaling receptors [91]. The genetic association between PPARG and endometriosis has been demonstrated [92]. Our study indicated that PPARG in the PPAR signaling pathway may be under the regulation of Jingshu granules for ovarian cyst treatment.

\section{Conclusion}

Our study reported multiactive ingredients in therapeutics by Jingshu granules to provide multitarget, multisignaling pathway regulation of ovarian cyst activity. The mechanism of action of Jingshu granules in ovarian cysts involved multiple active ingredients, targets, and signaling pathways. The systemic pharmacological analysis may provide a comprehensive understanding of the mechanisms of Jingshu granules in ovarian cysts. However, there is a limitation to our study. In vivo or in vitro experiments are lacking. Experimental verification of identified potential targets and signaling pathways of Jingshu granules in ovarian cysts is needed.

\section{Data Availability}

All data are available in the article.

\section{Conflicts of Interest}

The authors declare that there is no conflict of interest.

\section{Authors' Contributions}

Jili Xu and Yincong Xu contributed equally to this paper.

\section{References}

[1] E. J. Pavlik, F. R. Ueland, R. W. Miller et al., "Frequency and disposition of ovarian abnormalities followed with serial transvaginal ultrasonography," Obstetrics and gynecology, vol. 122, 2, PART 1, pp. 210-217, 2013.

[2] C. Dow, "Ovarian abnormalities in the bitch," Journal of comparative pathology, vol. 70, pp. 59-IN2, 1960.

[3] S. D. Johnston, M. V. R. Kustritz, and P. N. S. Olson, "Disorders of the canine ovary," Canine and Feline Theriogenology, pp. 193-205, 2001.

[4] M. Grant, D. Schlafer, M. Grant Maxie et al., Jubb, Kennedy, and Palmer's Pathology of Domestic Animals, Elsevier Health Sciences, 2007.

[5] E. Ignacio, R. Dua, S. Sarin et al., "Pelvic congestion syndrome: diagnosis and treatment," Seminars in Interventional Radiology, vol. 25, no. 4, pp. 361-368, 2008.

[6] Y. Knauf, H. Bostedt, K. Failing, S. Knauf, and A. Wehrend, "Gross pathology and endocrinology of ovarian cysts in bitches," Zuchthygiene, vol. 49, no. 3, pp. 463-468, 2014.

[7] K. Jeengar, V. Chaudhary, A. Kumar, S. Raiya, and G. N. Purohit, "Ovarian cysts in dairy cows: old and new concepts for definition, diagnosis and therapy," Animal Reproduction, vol. 11, pp. 63-73, 2014.

[8] M. Candiani, M. Barbieri, B. Bottani et al., "Ovarian recovery after laparoscopic enucleation of ovarian cysts: insights from echographic short-term postsurgical follow-up," Journal of Minimally Invasive Gynecology, vol. 12, no. 5, pp. 409-414, 2005.

[9] C. Z. Li, B. Liu, Z. Q. Wen, and Q. Sun, "The impact of electrocoagulation on ovarian reserve after laparoscopic excision of ovarian cysts: a prospective clinical study of 191 patients," Fertility and Sterility, vol. 92, no. 4, pp. 1428-1435, 2009.

[10] M. J. Mourits, E. G. de Vries, P. H. Willemse et al., "Ovarian cysts in women receiving tamoxifen for breast cancer," British Journal of Cancer, vol. 79, no. 11-12, pp. 1761-1764, 1999.

[11] M. Tafazoli, E. Fazeli, S. Dadgar, and M. Nematy, "The association of the dietary fat and functional ovarian cysts in women of reproductive age referring to three hospitals in Mashhad, Iran, 2014," International Journal of Community Based Nursing and Midwifery, vol. 4, no. 2, pp. 148-156, 2016.

[12] M. W. Beal, "Women's use of complementary and alternative therapies in reproductive health care," Journal of Nurse-Midwifery, vol. 43, no. 3, pp. 224-234, 1998.

[13] S. T. Huang and P. C. Chen, "Traditional Chinese medicine and infertility," Current Opinion in Obstetrics \& Gynecology, vol. 20, no. 3, pp. 211-215, 2008.

[14] S. C. Sze, H. P. Cheung, T. B. Ng et al., "Effects of Erxian decoction, a Chinese medicinal formulation, on serum lipid profile in a rat model of menopause," Chinese Medicine, vol. 6, no. 1, p. 40, 2011.

[15] S. Mohammad-Alizadeh-Charandabi, M. Shahnazi, J. Nahaee, and S. Bayatipayan, "Efficacy of black cohosh (Cimicifuga racemosa L.) in treating early symptoms of menopause: a randomized clinical trial," Chinese Medicine, vol. 8, no. 1, p. 20, 2013. 
[16] S. Wang, Y. Tong, T. B. Ng et al., "Network pharmacological identification of active compounds and potential actions of Erxian decoction in alleviating menopause-related symptoms," Chinese Medicine, vol. 10, no. 1, p. 19, 2015.

[17] X. Xu, Y. Tan, G. Jiang et al., "Effects of Bushen Tianjing recipe in a rat model of tripterygium glycoside-induced premature ovarian failure," Chinese Medicine, vol. 12, no. 1, p. 10, 2017.

[18] J. Ru, P. Li, J. Wang et al., "TCMSP: a database of systems pharmacology for drug discovery from herbal medicines," Journal of Cheminformatics, vol. 6, no. 1, p. 13, 2014.

[19] H. Yu, J. Chen, X. Xu et al., "A systematic prediction of multiple drug-target interactions from chemical, genomic, and pharmacological data," PLoS One, vol. 7, no. 5, article e37608, 2012.

[20] G. L. Mutter, J. Prat, and D. Schwartz, The female reproductive system, the peritoneum and pregnancy, pp. 847-922, 2012.

[21] P. Dharmshaktu, A. Kutiyal, and D. Dhanwal, "Vanishing large ovarian cyst with thyroxine therapy," Endocrinology, Diabetes \& Metabolism Case Reports, vol. 2013, article 130050, 2013.

[22] T. Perreault, R. Peet, and M. Watts, "Liberation ecologies: environment, development, Social Movements," Geographical Review, vol. 74, pp. 181-183, 1998.

[23] J. Iwamasa, S. Shibata, N. Tanaka, K. Matsuura, and H. Okamura, "The relationship between ovarian progesterone and proteolytic enzyme activity during ovulation in the gonadotropin-treated immature rat," Biology of Reproduction, vol. 46, no. 2, pp. 309-313, 1992.

[24] S. Liu, X. Zhang, C. Shi et al., "Altered microRNAs expression profiling in cumulus cells from patients with polycystic ovary syndrome," Journal of Translational Medicine, vol. 13, no. 1, 2015.

[25] A. P. Sobinoff, V. Pye, B. Nixon, S. D. Roman, and E. A. McLaughlin, "Adding insult to injury: effects of xenobioticinduced preantral ovotoxicity on ovarian development and oocyte fusibility," Toxicological Sciences, vol. 118, no. 2, pp. 653-666, 2010.

[26] E. T. Maizels, C. Joshua, J. C. R. Jones, and H. D. Mary, "Follicle stimulating hormone (FSH) activates the p38 mitogenactivated protein kinase pathway, inducing small heat shock protein phosphorylation and cell rounding in immature rat ovarian granulosa cells," Endocrinology, vol. 139, no. 7, pp. 3353-3356, 1998.

[27] Y. Q. Su, W. Karen, F. L. Pendola, M. J. O’Brien, and J. J. Eppig, "Mitogen-activated protein kinase activity in cumulus cells is essential for gonadotropin-induced oocyte meiotic resumption and cumulus expansion in the mouse," Endocrinology, vol. 143, no. 6, pp. 2221-2232, 2002.

[28] Y.-Q. Su, J. M. Denegre, K. Wigglesworth, F. L. Pendola, M. J. O'Brien, and J. J. Eppig, "Oocyte-dependent activation of mitogen-activated protein kinase (ERK1/2) in cumulus cells is required for the maturation of the mouse oocyte-cumulus cell complex," Developmental Biology, vol. 263, no. 1, pp. 126-138, 2003.

[29] S. Sela-Abramovich, E. Chorev, D. Galiani, and N. Dekel, "Mitogen-activated protein kinase mediates luteinizing hormone-induced breakdown of communication and oocyte maturation in rat ovarian follicles," Endocrinology, vol. 146, no. 3, pp. 1236-1244, 2005.

[30] M. Hunzicker-Dunn and E. T. Maizels, "FSH signaling pathways in immature granulosa cells that regulate target gene expression: branching out from protein kinase A," Cellular Signalling, vol. 18, no. 9, pp. 1351-1359, 2006.

[31] F. J. Diaz, M. J. O'Brien, K. Wigglesworth, and J. J. Eppig, "The preantral granulosa cell to cumulus cell transition in the mouse ovary: development of competence to undergo expansion," Developmental Biology, vol. 299, no. 1, pp. 91-104, 2006.

[32] T. Miyoshi, F. Otsuka, K. Inagaki et al., "Differential regulation of steroidogenesis by bone morphogenetic proteins in granulosa cells: involvement of extracellularly regulated kinase signaling and oocyte actions in follicle-stimulating hormoneinduced estrogen production," Endocrinology, vol. 148, no. 1, pp. 337-345, 2007.

[33] V. Sriraman, S. R. Modi, Y. Bodenburg, L. A. Denner, and R. J. Urban, "Identification of ERK and JNK as signaling mediators on protein kinase C activation in cultured granulosa cells," Molecular \& Cellular Endocrinology, vol. 294, no. 1-2, pp. 52-60, 2008.

[34] Z. Liu, H. Y. Fan, Y. Wang, and J. S. Richards, “Targeted disruption of Mapk14 (p38MAPKalpha) in granulosa cells and cumulus cells causes cell-specific changes in gene expression profiles that rescue COC expansion and maintain fertility," Molecular Endocrinology, vol. 24, no. 9, pp. 1794-1804, 2010.

[35] W. Xu, M. Tang, J. Wang, and L. Wang, "Identification of the active constituents and significant pathways of Cangfu Daotan decoction for the treatment of PCOS based on network pharmacology," Evidence-Based Complementary and Alternative Medicine, vol. 2020, Article ID 4086864, 15 pages, 2020.

[36] M. Kanatsu-Shinohara, R. M. Schultz, and G. S. Kopf, “Acquisition of meiotic competence in mouse oocytes: absolute amounts of p34(cdc2), cyclin B1, cdc25C, and wee1 in meiotically incompetent and competent oocytes," Biology of Reproduction, vol. 63, no. 6, pp. 1610-1616, 2000.

[37] W. Mak, J. Xia, E. C. Cheng, K. Lowther, and H. Lin, “A role of Pumilio 1 in mammalian oocyte maturation and maternal phase of embryogenesis," Cell \& Bioscience, vol. 8, no. 1, 2018.

[38] A. N. Pandey and S. K. Chaube, "Reduction of nitric oxide level leads to spontaneous resumption of meiosis in diplotenearrested rat oocytes cultured in vitro," Experimental Biology and Medicine, vol. 240, no. 1, pp. 15-25, 2015.

[39] S. Prasad, M. Tiwari, B. Koch, and S. K. Chaube, "Morphological, cellular and molecular changes during postovulatory egg aging in mammals," Journal of Biomedical Science, vol. 22, no. 1, 2015.

[40] D. Adhikari, W. Zheng, Y. Shen et al., "Cdk1, but not Cdk2, is the sole Cdk that is essential and sufficient to drive resumption of meiosis in mouse oocytes," Human Molecular Genetics., vol. 21, no. 11, pp. 2476-2484, 2012.

[41] D. J. Haisenleder, A. C. Dalkin, G. A. Ortolano, J. C. Marshall, and M. A. Shupnik, "A pulsatile gonadotropin-releasing hormone stimulus is required to increase transcription of the gonadotropin subunit genes: evidence for differential regulation of transcription by pulse frequency in vivo," Endocrinology, vol. 128, no. 1, pp. 509-517, 1991.

[42] D. S. Mistry, R. Tsutsumi, M. Fernandez et al., "Gonadotropinreleasing hormone pulse sensitivity of follicle-stimulating hormone-beta gene is mediated by differential expression of positive regulatory activator protein 1 factors and corepressors SKIL and TGIF1," Molecular Endocrinology, vol. 25, no. 8, pp. 1387-1403, 2011.

[43] H. A. Ferris and M. A. Shupnik, "Mechanisms for pulsatile regulation of the gonadotropin subunit genes by GNRH1," Biology of Reproduction, vol. 74, no. 6, pp. 993-998, 2006. 
[44] C. T. B. J. Waegemaekers, A. V. D. Berg, A. Blankhart, F. M. Helmerhorst, and N. Naaktgeboren, "Ovarian cyst formation during the use of GnRH analogue in an IVF programme," Acta Europaea Fertilitatis, vol. 18, p. 411, 1988.

[45] L. L. Lindaman, D. M. Yeh, C. Xie, K. M. Breen, and D. Coss, "Phosphorylation of ATF2 and interaction with NFY induces c-Jun in the gonadotrope," Molecular \& Cellular Endocrinology, vol. 365, no. 2, pp. 316-326, 2013.

[46] K. G. Geles, R. N. Freiman, W.-L. Liu, S. Zheng, E. Voronina, and R. Tjian, "Cell-type-selective induction of c-jun by TAF4b directs ovarian-specific transcription networks," Proceedings of the National Academy of Sciences of the United States of America, vol. 103, no. 8, pp. 2594-2599, 2006.

[47] S. B. Serizier and K. McCall, "Scrambled eggs: apoptotic cell clearance by non-professional phagocytes in the Drosophila ovary," Frontiers in Immunology, vol. 8, p. 1642, 2017.

[48] Q. Zheng, Y. Li, D. Zhang et al., "ANP promotes proliferation and inhibits apoptosis of ovarian granulosa cells by NPRA/PGRMC1/EGFR complex and improves ovary functions of PCOS rats," Cell Death \& Disease, vol. 8, no. 10, article e3145, 2017.

[49] A. Bukovsky, M. R. Caudle, J. A. Keenan et al., "Association of mesenchymal cells and immunoglobulins with differentiating epithelial cells," BMC Developmental Biology, vol. 1, no. 1, p. 11, 2001.

[50] A. Bukovský, J. A. Keenan, M. R. Caudle, J. Wimalasena, N. B. Upadhyaya, and S. E. Van Meter, "Immunohistochemical studies of the adult human ovary: possible contribution of immune and epithelial factors to folliculogenesis," American Journal of Reproductive Immunology, vol. 33, pp. 323-340, 1995.

[51] A. Bukovsky, M. R. Caudle, M. Svetlikova, J. Wimalasena, M. E. Ayala, and R. Dominguez, "Oogenesis in adult mammals, including humans: A Review," Endocrine, vol. 26, no. 3, pp. 301-316, 2005.

[52] J. C. Chapman, S. H. Min, S. M. Freeh, and S. D. Michael, “The estrogen-injected female mouse: new insight into the etiology of PCOS," Reproductive Biology and Endocrinology, vol. 7, no. $1,2009$.

[53] J. A. S. Richards, Z. Liu, and M. Shimada, "Immune-like mechanisms in ovulation," Trends in Endocrinology \& Metabolism, vol. 19, no. 6, pp. 191-196, 2008.

[54] C. Hernandes, B. Y. Gueuvoghlanian-Silva, V. U. Monnaka, N. M. Ribeiro, W. de Oliveira Pereira, and S. Podgaec, "Regulatory $\mathrm{T}$ cells isolated from endometriotic peritoneal fluid express a different number of Toll-like receptors," Einstein (São Paulo), vol. 18, 2020.

[55] D. Joyce, C. Albanese, J. Steer, M. Fu, B. Bouzahzah, and R. G. Pestell, "NF- $\kappa \mathrm{B}$ and cell-cycle regulation: the cyclin connection," Cytokine \& Growth Factor Reviews, vol. 12, no. 1, pp. 73-90, 2001.

[56] S. A. Schwartz, A. Hernandez, and B. M. Evers, "The role of NF- $\kappa \mathrm{B} / \mathrm{I} \kappa \mathrm{B}$ proteins in cancer: implications for novel treatment strategies," Surgical Oncology, vol. 8, no. 3, pp. 143-153, 1999.

[57] M. Conti, M. Hsieh, J.-Y. Park, and Y.-Q. Su, "Role of the EGF network in ovarian follicles," Molecular Endocrinology, vol. 20, pp. 715-723, 2006.

[58] M. Shimada, I. Hernandez-Gonzalez, I. Gonzalez-Robayna, and J. A. S. Richards, "Paracrine and autocrine regulation of epidermal growth factor-like factors in cumulus oocyte com- plexes and granulosa cells: key roles for prostaglandin synthase 2 and progesterone receptor," Molecular Endocrinology., vol. 20, no. 6, pp. 1352-1365, 2006.

[59] H.-Y. Fan, Z. Liu, M. Shimada et al., "MAPK3/1 (ERK1/2) in ovarian granulosa cells are essential for female fertility," Science, vol. 324, pp. 938-941, 2009.

[60] N. Filigheddu, I. Gregnanin, P. E. Porporato et al., "Differential expression of microRNAs between eutopic and ectopic endometrium in ovarian endometriosis," Journal of Biomedicine \& Biotechnology, vol. 2010, Article ID 369549, 29 pages, 2010.

[61] Y. Y. Da Li, F.-F. Bi, T.-N. Zhang et al., "Autophagy is activated in the ovarian tissue of polycystic ovary syndrome," Reproduction, vol. 155, no. 1, pp. 85-92, 2018.

[62] B. Pothuri, M. M. Leitao, D. A. Levine et al., "Genetic analysis of the early natural history of epithelial ovarian carcinoma," PLoS One, vol. 5, no. 4, article e10358, 2010.

[63] X.-X. Wang, J.-Z. Wei, J. Jiao, S.-Y. Jiang, D.-H. Yu, and D. Li, "Genome-wide DNA methylation and gene expression patterns provide insight into polycystic ovary syndrome development," Oncotarget, vol. 5, no. 16, pp. 6603-6610, 2014.

[64] P. Sagvekar, P. Kumar, V. Mangoli, S. Desai, and S. Mukherjee, "DNA methylome profiling of granulosa cells reveals altered methylation in genes regulating vital ovarian functions in polycystic ovary syndrome," Clinical Epigenetics, vol. 11, no. 1, p. 61, 2019.

[65] K. Nakao, H. Kishi, F. Imai, H. Suwa, T. Hirakawa, and T. Minegishi, "TNF- $\alpha$ suppressed FSH-induced LH receptor expression through transcriptional regulation in rat granulosa cells," Endocrinology, vol. 156, no. 9, pp. 3192-3202, 2015.

[66] O. Sipak-Szmigiel, P. Włodarski, E. Ronin-Walknowska et al., "Serum and peritoneal fluid concentrations of soluble human leukocyte antigen, tumor necrosis factor alpha and interleukin 10 in patients with selected ovarian pathologies," Journal of Ovarian Research, vol. 10, no. 1, p. 25, 2017.

[67] Z. Liu, M. D. Rudd, I. Hernandez-Gonzalez et al., "FSH and FOXO1 regulate genes in the sterol/steroid and lipid biosynthetic pathways in granulosa cells," Molecular Endocrinology, vol. 23, no. 5, pp. 649-661, 2009.

[68] C. J. Brewer and A. H. Balen, "The adverse effects of obesity on conception and implantation," Reproduction, vol. 140, no. 3, pp. 347-364, 2010.

[69] L. Cui, H. Zhao, B. Zhang et al., "Genotype-phenotype correlations of PCOS susceptibility SNPs identified by GWAS in a large cohort of Han Chinese women," Human Reproduction, vol. 28, no. 2, pp. 538-544, 2013.

[70] V. R. Tumu, S. Govatati, P. Guruvaiah, M. Deenadayal, S. Shivaji, and M. Bhanoori, "An interleukin-6 gene promoter polymorphism is associated with polycystic ovary syndrome in South Indian women," Journal of Assisted Reproduction \& Genetics, vol. 30, no. 12, pp. 1541-1546, 2013.

[71] S. Radavelli-Bagatini, I. O. de Oliveira, R. B. Ramos et al., "Haplotype TGTG from SNP 45T/G and 276G/T of the adiponectin gene contributes to risk of polycystic ovary syndrome," Journal of Endocrinological Investigation, vol. 36, no. 7, pp. 497-502, 2013.

[72] E. Nilsson, B. Zhang, and M. K. Skinner, "Gene bionetworks that regulate ovarian primordial follicle assembly," $B M C$ Genomics, vol. 14, no. 1, p. 496, 2013.

[73] S. Johanna, W. Birgitta, M. A. Lis, L. Svend, N. Lars, and B. Mats, "Differential expression of inflammation-related genes in the ovarian stroma and granulosa cells of PCOS 
women," Molecular Human Reproduction, vol. 20, no. 1, pp. 49-58, 2013.

[74] H. Jiang, Z. Xing, W. Lu, Z. Qian, H. Yu, and J. Li, "Transcriptome analysis of red swamp crawfish Procambarus clarkii reveals genes involved in gonadal development," PLoS One, vol. 9, no. 8, article e105122, 2014.

[75] B. R. Calvi, M. A. Lilly, and A. C. Spradling, "Cell cycle control of chorion gene amplification," Genes \& Development, vol. 12, no. 5, pp. 734-744, 1998.

[76] A. Satyanarayana and P. Kaldis, "Mammalian cell-cycle regulation: several Cdks, numerous cyclins and diverse compensatory mechanisms," Oncogene, vol. 28, no. 33, pp. 2925-2939, 2009.

[77] J. Peretz, S. L. Neese, and J. A. Flaws, "Mouse strain does not influence the overall effects of bisphenol A-induced toxicity in adult antral follicles," Biology of reproduction, vol. 89, no. 5, p. 108, 2013.

[78] D. S. Moons, S. Jirawatnotai, T. Tsutsui et al., "Intact follicular maturation and defective luteal function in mice deficient for cyclin- dependent kinase-4," Endocrinology, vol. 143, no. 2, pp. 647-654, 2002.

[79] D. R. Meldrum, "Vascular endothelial growth factor, polycystic ovary syndrome, and ovarian hyperstimulation syndrome," Fertility \& Sterility, vol. 78, pp. 1170-1171, 2003.

[80] B. R. Kamat, L. F. Brown, E. J. Manseau, D. R. Senger, and H. F. Dvorak, "Expression of vascular permeability factor/vascular endothelial growth factor by human granulosa and theca lutein cells. Role in corpus luteum development," The American journal of pathology., vol. 146, no. 1, pp. 157-165, 1995.

[81] L. Karimzadeh, M. Nabiuni, A. Sheikholeslami, and S. Irian, "Bee venom treatment reduced C-reactive protein and improved follicle quality in a rat model of estradiol valerateinduced polycystic ovarian syndrome," Journal of Venomous Animals and Toxins including Tropical Diseases, vol. 18, no. 4, pp. 384-392, 2012.

[82] J. Greenaway, K. Connor, H. G. Pedersen, B. L. Coomber, J. LaMarre, and J. Petrik, "Vascular endothelial growth factor and its receptor, Flk-1/KDR, are cytoprotective in the extravascular compartment of the ovarian follicle," Endocrinology, vol. 145, no. 6, pp. 2896-2905, 2004.

[83] K. Lin, J. Ma, R. Wu, C. Zhou, and J. Lin, "Influence of ovarian endometrioma on expression of steroid receptor RNA activator, estrogen receptors, vascular endothelial growth factor, and thrombospondin 1 in the surrounding ovarian tissues," Reproductive Sciences, vol. 21, no. 2, pp. 183-189, 2013.

[84] H.-Y. Fan, Z. Liu, P. F. Johnson, and J. A. S. Richards, “CCAA T/enhancer-binding proteins (C/EBP)- $\alpha$ and $-\beta$ are essential for ovulation, luteinization, and the expression of key target genes," Molecular Endocrinology, vol. 25, pp. 253-268, 2011.

[85] S. Panigone, M. Hsieh, M. Fu, L. Persani, and M. Conti, "Luteinizing hormone signaling in preovulatory follicles involves early activation of the epidermal growth factor receptor pathway," Molecular Endocrinology, vol. 22, no. 4, pp. 924936, 2008.

[86] Y. Wang, X. Fu, J. Xu, Q. Wang, and H. Kuang, "Systems pharmacology to investigate the interaction of berberine and other drugs in treating polycystic ovary syndrome," Scientific Reports, vol. 6, no. 1, article 28089, 2016.

[87] W. Zheng, J. Wu, J. Gu et al., "Modular characteristics and mechanism of action of herbs for endometriosis treatment in Chinese medicine: a data mining and network pharmacology-based identification," Frontiers in pharmacology., vol. 11, p. 147, 2020.

[88] C. M. Komar, "Peroxisome proliferator-activated receptors (PPARs) and ovarian function-implications for regulating steroidogenesis, differentiation, and tissue remodeling," Reproductive Biology and Endocrinology, vol. 3, no. 1, p. 41, 2005.

[89] M. Zaree, V. Shahnazi, S. Fayezi et al., "Expression levels of PPAR $\gamma$ and CYP-19 in polycystic ovarian syndrome primary granulosa cells: influence of $\omega$-3 fatty acid," International Journal of Fertility \& Sterility, vol. 9, no. 2, pp. 197-204, 2015.

[90] D. Toms, S. Xu, B. Pan, D. Wu, and J. Li, "Progesterone receptor expression in granulosa cells is suppressed by microRNA378-3p," Molecular and Cellular Endocrinology, vol. 399, pp. 95-102, 2015.

[91] A. Bonnet, C. Cabau, O. Bouchez et al., "An overview of gene expression dynamics during early ovarian folliculogenesis: specificity of follicular compartments and bi-directional dialog," BMC Genomics, vol. 14, no. 1, p. 904, 2013.

[92] G. W. Montgomery, D. R. Nyholt, Z. Z. Zhao et al., "The search for genes contributing to endometriosis risk," Human Reproduction Update., vol. 14, no. 5, pp. 447-457, 2008. 